\title{
On the Point of Emergence of a Microwave Beam Entering a Linearly Graded Plasma
}

\author{
A. L. Cullen \\ Contribution From the Department of Electronic and Electrical Engineering, University of Sheffield, England
}

(Received August 31, 1964)

\begin{abstract}
A beam of microwave radiation is assumed to enter a linearly graded plane-stratified plasma. If the electron density reaches the critical value $\omega^{2} \epsilon_{0} m / e^{2}$ at a distance $x_{c}$ from the plasma boundary, ray theory predicts that the beam will emerge from the plasma at a distance $2 x_{c} \sin 2 \theta$ from its point of entry, $\theta$ being the angle of incidence. The validity of this result is studied using wave theory.
\end{abstract}

\section{Introduction}

The electron-density profile in a plasma has been studied by Kharadly [1963] using a microwave beam obliquely incident on the plasma boundary. By studying the dependence of the position at which the beam emerges again from the plasma as a function of the angle of incidence, the electron-density profile can be calculated. The calculation assumes that ray theory is valid, and it is the purpose of the present paper to study the validity of ray theory for the special case of a linear variation of electron density with position. The ray theory result is very simple; the distance between the entering and emerging rays is $2 x_{c}$ sin $2 \theta$, where $x_{c}$ is the depth in the plasma at which the electron density reaches the critical value at which the refractive index is zero, and $\theta$ is the angle of incidence.

\section{Wave Theory of Reflection}

The propagation of electromagnetic waves in stratified plasmas has received a great deal of attention in the literature, and the resulting body of knowledge has recently been summarized very conveniently by Budden [1961], Wait [1962], and Ginzburg [1964].

In particular, it is well known that electromagnetic wave propagation in a linearly graded plasma can be expressed in terms of Airy functions [e.g., Wait, 1962]. It is therefore possible to obtain an exact solution in closed form for the reflection coefficient for a plane wave in free space incident on such a plasma. When the electric field is perpendicular to the plane of incidence, the reflection coefficient, using Budden's notation [1961], is

$$
\frac{\cos \theta A i\left(\zeta_{0}\right)-j\left(\frac{1}{k x_{c}}\right)^{1 / 3} A i^{\prime}\left(\zeta_{0}\right)}{\cos \theta A i\left(\zeta_{0}\right)+j\left(\frac{1}{k x_{c}}\right)^{1 / 3} A i^{\prime}\left(\zeta_{0}\right)} .
$$

In the absence of collisions, $k$ and $\zeta_{0}$ are real numbers, so $|R|=1$, and we can write $R=e^{j \psi}$, where

$$
\tan \frac{\psi}{2}=-\frac{1}{\cos \theta}\left(\frac{1}{k x_{c}}\right)^{1 / 3} \frac{A i^{\prime}\left(\zeta_{0}\right)}{A i\left(\zeta_{0}\right)}=f(\theta),
$$

say, and

$$
\zeta_{0}=-\cos ^{2} \theta\left(k x_{c}\right)^{2 / 3}
$$

If we consider a narrow angular spectrum of plane waves incident on the plasma, the reflected spectrum will be modified because of the dependence of $\psi$ on $\theta$ through (2) and (3).

We wish to find the position at which the electric field of the reflected wave has a maximum value; let $\Delta y$ be the distance (measured along the plasma boundary) between this position and the corresponding position for the incident wave. The principle of stationary phase can be invoked to show that

$$
\Delta y=\frac{1}{k \cos \theta} \cdot \frac{d \psi}{d \theta}
$$

Using (2), we can write

$$
\frac{d \psi}{d \theta}=\frac{2 f^{\prime}(\theta)}{1+f^{2}(\theta)}
$$

The denominator of this expression can be written in terms of the Airy functions thus

$$
1+f^{2}(\theta)=\frac{1}{\zeta_{0}}\left\{\zeta_{0}-\frac{A i^{\prime}\left(\zeta_{0}\right)^{2}}{A i\left(\zeta_{0}\right)^{2}}\right\}
$$

The calculation of the derivative $f^{\prime}(\theta)$ is straightforward, but is simplified considerably by using the result $A i^{\prime \prime}\left(\zeta_{0}\right)=\zeta_{0} A i\left(\zeta_{0}\right)$ which follows from the differential equation which the Airy functions satisfy. Thus, we get 


$$
\begin{array}{r}
f^{\prime}(\theta)=-\left(k x_{c}\right)^{-1 / 3}\left[\frac{\sin \theta}{\cos ^{2} \theta} \frac{A i^{\prime}\left(\zeta_{0}\right)}{A i\left(\zeta_{0}\right)}+2 \sin \theta\left(k x_{c}\right)^{2 / 3}\right. \\
\left.\left\{\zeta_{0}-\frac{A i^{\prime}\left(\zeta_{0}\right)^{2}}{A i\left(\zeta_{0}\right)^{2}}\right\}\right]
\end{array}
$$

Combining (4), (5), (6), and (7), and rearranging the resulting expression with the help of (3), we finally get

$$
\Delta y=2 x_{c} \sin 2 \theta\left[1-\frac{1}{-2 \zeta_{0}\left\{-\zeta_{0} \frac{A i\left(\zeta_{0}\right)}{A i^{\prime}\left(\zeta_{0}\right)}+\frac{A i^{\prime}\left(\zeta_{0}\right)}{A i\left(\zeta_{0}\right)}\right\}}\right]
$$

It is convenient to use a parameter $\varphi$ rather than $\zeta_{0}$, where

$$
\varphi=\frac{2}{3}\left|\zeta_{0}\right|^{3 / 2}=\frac{2}{3} k x_{c} \cos ^{3} \theta .
$$

We can then define a correction factor $F(\phi)$ by the following equation:

$$
\Delta y=2 x_{c} \sin 2 \theta \cdot F(\varphi),
$$

$F(\varphi)$ is therefore the factor by which the raytheory result must be multiplied to get the wavetheory result.

\section{Asymptotic Formulas}

If (1) is simplified by using the first terms in the asymptotic expansions of $A i$ and $A i^{\prime}$, the reflection coefficient becomes

$$
R=j \exp \left\{-\frac{4}{3} j \cos ^{3} \theta k x_{c}\right\}
$$

so that

$$
\psi=-\frac{4}{3} k x_{c} \cos ^{3} \theta
$$

When (11) is substituted in (4), the ray-theory result is again obtained, thus verifying the wellknown between ray theory and the W.K.B. approximation. If the first two terms in each asymptotic series are retained, a first order correction to ray theory can be found, in terms of elementary functions and is as follows:

$$
F(\varphi) \simeq \frac{\frac{31}{3}+64 \phi^{2}+\left(\frac{63}{3}+64 \phi^{2}\right) \tan ^{2} \Phi}{\left(8 \phi+\frac{7}{3} \tan \Phi\right)^{2}+(8 \phi \tan \Phi-1)^{2}}
$$

where $\Phi=\varphi-\pi / 4$. There is, however, little to be gained from the use of (12) rather than (8) as the Airy functions are quite adequately tabulated.

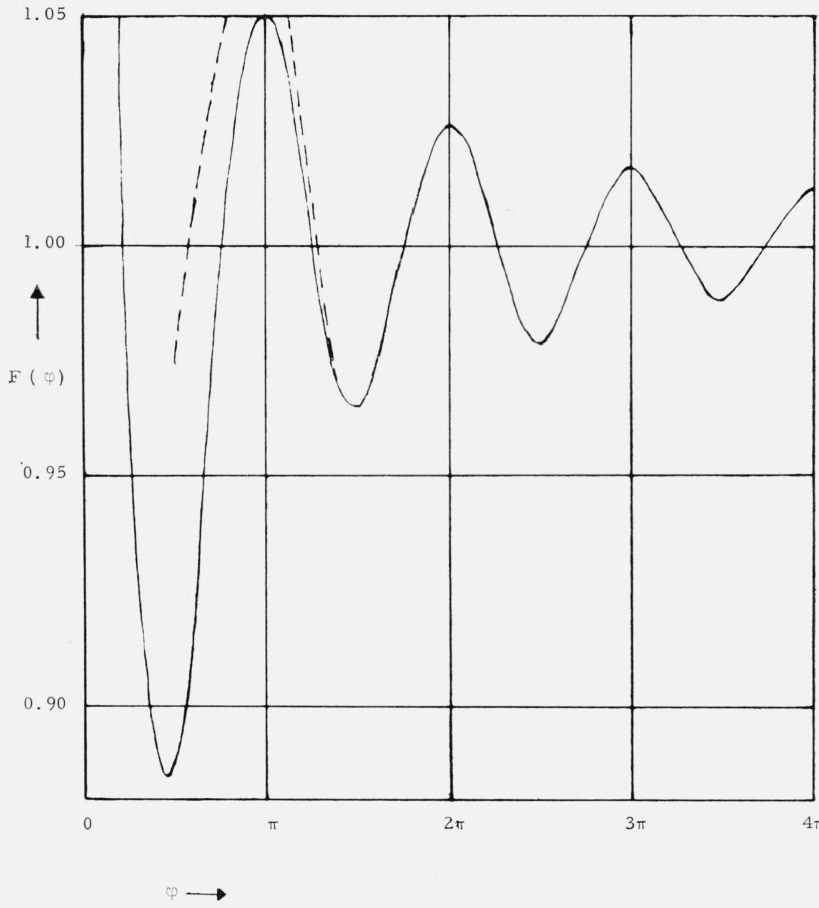

Figure 1. Ray theory correction factor.

\section{Discussion of Results}

In figure 1 the function $F(\varphi)$ is plotted as a function of $\varphi$. The full line is the exact expression in (8); the broken line is obtained from (12) and shows how the asymptotic result fails for $\phi<3 \pi / 2$. The Airy functions $A i$ and $A i^{\prime}$ are tabulated [British Association Mathematical Tables, 1946].

It is clear that if $\varphi>0.65 \pi$, ray theory and wave theory agree to within 5 percent. For example, if $\theta=60^{\circ}$, we must have $x_{c}>3.9 \lambda$ for 5 percent accuracy. This condition is quite easily met in Kharadly's experiments, but it is important to bear this limitation of ray theory constantly in mind in applying the oblique incidence plasma diagnostic technique in the millimeter-wave region. A similar correction factor occurs in correcting ray theory in the vicinity of caustics [e.g., Wait, 1962, p. 338].

\section{References}

British Association Mathematical Tables (1946), The airy integral, Part-Vol. B (Cambridge University Press, Cambridge).

Budden, K. G. (1961), Radio waves in the ionosphere (Cambridge University Press, Cambridge).

Ginzburg, V. L. (1964), The propagation of electromagnetic waves in plasmas (Pergamon Press, Oxford and Macmillan and Co., New York, N.Y.).

Kharadly, M. M. Z. (1963), A new millimetre-wave method for determination of electron-density profiles in a linear discharge, Proc. IEE 110, No. 7, 1202.

Wait, J. R. (1962), Electromagnetic waves in stratified media (Pergamon Press, Oxford and Macmillan and Co., New York, N.Y.).

(Paper 69D2-452) 